\title{
Perfused hypertonic-saline-augmented needle enlarges ablation zones in ex vivo porcine livers
}

\author{
CAOYE WANG, QI WANG, DACHUAN ZHANG, ZHONGMING HE and WENHUA CHEN \\ Department of Interventional Radiology, Changzhou First People's Hospital, Changzhou, Jiangsu 213003, P.R. China
}

Received May 17, 2016; Accepted July 20, 2017

DOI: $10.3892 / \mathrm{ol} .2017 .7352$

\begin{abstract}
There is a great clinical requirement to improve radiofrequency ablation (RFA) efficacy and create larger coagulation necrotic areas. The aim of the present study was to assess the ability of a hypertonic-saline (HS)-enhanced multipolar RFA technique using a perfused electrode to increase RF-created coagulation necrosis, and to compare that technique with natural saline-augmented needle and conventional multipolar RFA. A total of 18 ablations were performed in explanted porcine livers. A total of 6 thermal ablation zones were created in each of 3 groups treated with the conventional multipolar mode, the multipolar mode with $0.9 \% \mathrm{NaCl}$ and the multipolar mode with $6 \%$ $\mathrm{NaCl}$, respectively. During RFA, the dimensions and volumes of the ablation zones were compared, and gross and microscopic pathological evaluations were performed. Multipolar RFA with $6 \% \mathrm{NaCl}$ created the largest short-axis diameters and volumes of coagulation necrosis $\left(3.89 \pm 0.09 \mathrm{~mm}\right.$ and $40.01 \pm 2.86 \mathrm{~mm}^{3}$, respectively) among the three groups (conventional group: $2.31 \pm 0.04 \mathrm{~mm}$ and $8.99 \pm 0.52 \mathrm{~mm}^{3}$, respectively; $0.9 \% \mathrm{NaCl}$ solution group: $3.17 \pm 0.05 \mathrm{~mm}$ and $21.79 \pm 1.05 \mathrm{~mm}^{3}$, respectively). Overall, multipolar RFA with the instillation of $6 \%$ $\mathrm{NaCl}$ solution through an open perfusion system created a larger ablation zone compared with the conventional and $0.9 \%$ $\mathrm{NaCl}$ modes. Therefore, HS-enhanced multipolar RFA may be a promising approach for treating large liver tumors.
\end{abstract}

\section{Introduction}

Radiofrequency ablation (RFA) has been validated as a safe and efficient treatment for hepatocellular carcinoma (HCC) and focal metastatic liver tumors in patients who are not candidates for hepatic resection or who refuse surgery (1). However, clinical studies have identified a high rate of unsuccessful RFA treatment for large tumors (diameter $>3 \mathrm{~cm}$ ) and also for local tumors with

Correspondence to: Dr Qi Wang, Department of Interventional Radiology, Changzhou First People's Hospital, Juqian Road No.185, Changzhou, Jiangsu 213003, P.R. China

E-mail: caoyewang@139.com

Key words: liver radiofrequency ablation, sodium chloride, porcine liver, hypertonic-saline-enhanced electrodes a high recurrence rate (2). A typical lesion created by a linear RFA ablation probe may be modeled as a three-dimensional spheroid, which has one major and two minor axes, with the major axis lying parallel to the probe shaft. The extent of the long axis of the lesion may be controlled by selecting different lengths of electrodes, but controlling the short axis remains an issue that limits the success of ablation. A number of strategies currently exist for overcoming this limitation and increasing the ablated volume. Firstly, the electrode surface is enhanced by increasing the probe gauge (3), using several applicators in a cluster (4), a multiprobe array (5) and expandable applicators (6). Secondly, the power output may be increased and combined with strategies for reducing probe heating, such as pulsing the energy application (7). Thirdly, energy application may be affected at the interface between the applicator and tissue by preventing tissue dehydration and carbonization, using actively cooled applicators with closed circuit water cooling (8), perfusion (9) or cryogenic cooling (10). Studies have demonstrated that saline or hypertonic saline (HS)-enhanced bipolar RFA creates larger lesions compared with monopolar RFA, due to the high electrical conductivity of saline (11-15). However, studies assessing the association between the concentration of the perfused $\mathrm{NaCl}$ solution and the diameter of the coagulation necrosis caused by ablation are scarce (16). There has been little comparative data to validate these results on tissue coagulation. Concurrently, there is a large clinical demand to improve RFA efficacy to create larger coagulation necrosis areas $(17,18)$.

In the present study, increasing the electrode surface (multiprobearray) and continuous infusion ofelectrical conductivity fluid during RFA were combined to enlarge the ablation volume. The sizes, shapes and pathology data of the ablated lesions created by an internally cool-tip electrode multipolar RFA applicator with a perfused HS-augmented and normal saline (NS)-augmented needles, and conventional internally cool-tip electrode multipolar RF, were compared. The results of the present study demonstrated that using perfused hypertonic-saline-augmented needle combined with the RFA instrument may enlarge the ablation zones and improve the efficacy for treatment of large tumors.

\section{Materials and methods}

RFA system. An RF generator (Radionics Inc., Burlington, MA, USA) capable of producing $200 \mathrm{~W}$ was used with a 21-gauge needle (21Gx200 mm; Hakko Co., Ltd., Nagano, Japan) with a 3-cm exposed-tip internally water-cooled electrode [multipolar 
(three applicators) Cool-tip ${ }^{\mathrm{TM}}$ RFA Cluster Electrode kit; Covidien IIc, Mansfield, MA, USA] (Fig. 1). Needle cooling was achieved by a continuous internal perfusion of the applicator (Microinfusion pump WZS-50F2; Zhejiang Medical University Equipment Co., Ltd, Hangzhou, China). A mechanical pump (Radionics Cool-tip RF system; Radionics Inc.) was used to perfuse the electrode with $0^{\circ} \mathrm{C}$ saline solution at a rate of $110 \mathrm{ml} / \mathrm{min}$ in a closed perfusion circuit prior to each ablation, in order to cool the electrode tip and reduce the charring of the surrounding tissue that may decrease tissue conductivity and block RF energy. The generator was used in impedance control mode delivering a pulsed RF application. RF was applied to each electrode for $15 \mathrm{~min}$ under $50-\mathrm{W}$ conditions. A total of 3 ablation zones were created under each condition. A total of 18 ablations were performed. In the experiments of the present study, in case of a fast increase of impedance, if the tissue impedance rose $10 \Omega$ beyond the baseline value $(60 \Omega)$, the current switched off automatically for $15 \mathrm{sec}$. Auto-regulation of the generator was current-based and impedance-controlled; full impedance was defined as the point when the generator automatically sensed that the resistance was too high, and power was no longer deposited efficiently in the tissues. At this point, the generator was turned off. Following a pause of $15 \mathrm{sec}$, the system was switched on again.

\section{RFA protocol}

Conventional RFA group. Lesions were created in fresh (within $1 \mathrm{~h}$ of mortality) room-temperature swine livers obtained from the local butcher. The room-temperature livers were positioned on a $10-\mathrm{cm}^{2}$ grounding pad that was placed at least $30 \mathrm{~cm}$ away from the electrode. The electrodes were placed $3 \mathrm{~cm}$ into the livers.

Perfused NS and HS-augmented RFA groups. The experiments were performed in the same manner as the conventional RFA group, with the exception of a 21-gauge open perfused electrode located in the middle of the needle being placed into the porcine livers; the $\mathrm{NaCl}$ solution was instilled into the tissue through the electrode, with an injection rate of $60 \mathrm{ml} / \mathrm{h}$. The concentrations of the $\mathrm{NaCl}$ solutions perfused were 0.9 and $6 \%$ in the NS and HS-augmented RFA groups, respectively (16).

Evaluating the size and shape of ablation lesions. The dimensions of the ablation zones were compared among the groups. The ablated lesions exhibited an elliptical shape and were well-demarcated within the normal liver tissue. The ablated lesions were evaluated macroscopically and histologically. Dimensions of the lesions were compared among the conventional-RFA, $0.9 \%$ NS-RFA and 6\% HS-augmented-RFA groups. Each post-RF ablation coagulation specimen was cut along the long axis of the coagulation (ablation lesion along the axis of the electrode or needle insertion); therefore, only the length and one diameter $\left(90^{\circ}\right.$ to the electrode or needle) measurement were utilized and measured with a ruler by two investigators who reached consensus on each dimension. The length and each diameter of the white central zone of necrosis were measured with a ruler to within $1 \mathrm{~mm}$. It was not possible to measure the other diameter. However, in a pre-experiment, a separate section of tissue along the other axis was measured, and it was identified that the diameters were usually symmetrical in this ex vivo experiment. Therefore, the single diameter was used twice in volume calculations. The volume of the ablation lesion was calculated by approximating it to an ellipsoid using the following formula: $V=1 / 6 \times \pi \times \mathrm{LD} \times \mathrm{TD}^{2}$, where $\mathrm{V}$ is volume, $\pi$ is the circumference of a circle divided by its diameter, LD is longitudinal diameter and TD is transverse diameter $(9,18)$. The representative tissue specimens of each ablation zone containing the central area of necrosis and the transition zone towards the healthy liver tissue were selected. Representative tissue was maintained in $10 \%$ formaldehyde solution at $28^{\circ} \mathrm{C}$ for $24 \mathrm{~h}$. Following dehydration with $70 \%$ xylene and $100 \%$ absolute ethanol (1:1), tissue was embedded in paraffin for $130 \mathrm{~min}$ at $58^{\circ} \mathrm{C}$. Histological features were assessed from $4-\mu \mathrm{m}$ thick slides with hematoxylin and eosin (H\&E) staining $\left(1 \% \mathrm{H} \& \mathrm{E}, 25 \mathrm{~min}, 26^{\circ} \mathrm{C}\right)$ under light microscopy (magnifications, $\mathrm{x} 40, \mathrm{x} 200$ and $\mathrm{x} 400$ ).

Statistical analysis. Data are presented as the mean \pm standard deviation. Ablation lesion dimensions were compared among conventional RFA, NS-RFA and $6 \%$ perfused HS-augmented RFA with the Mann Whitney U-test with Bonferroni correction for several independent samples using SPSS 15 (SPSS Inc., Chicago, IL, USA). A two-sided P-value of $<0.05$ was considered to indicate a statistically significant difference.

\section{Results}

Macroscopic data. All procedures were successful, and 18 lesions were created by conventional-RFA, NS-RFA and $6 \%$ perfused HS-augmented RFA. Subsequent to slicing, all ablation zones macroscopically demonstrated a black-brown-yellow appearance in their center corresponding to the necrotic ablation, clearly demarcated by a red hyperemic rim (Fig. 2).

The conventional-RFA lesions appeared elliptical in shape and were well-demarcated within the normal liver tissue. There were central cavities representing the needle tract surrounded by pale coagulation necrosis, and the outer coagulation necrosis appeared as a brownish color (Fig. 2A).

The NS-RFA lesions were approximately elliptical in shape and were in well-demarcated areas within the liver tissue. The post-RFA liver surface has four poles. The outer three poles corresponded to the RFA probes and the middle pole was used to inject $0.9 \% \mathrm{NaCl}$ liquid. The central zone, whose diameter was slightly larger compared with the channel, was a hollow space created by the vaporization of tissue and withdrawal of the channel. Beyond the central area was a region of brown coagulated tissues, and reddish tissue was in the outermost region of the lesion, which was bordered by normal liver tissue (Fig. 2B).

By contrast, 6\% perfused HS-augmented RFA lesions were also elliptical in shape, but the central portion was jelly-like, and the track of the RF electrode was clearer. Surrounding the jelly-like tissue was off-white or dust-colored coagulation necrosis, and beyond this was an area of reddish coagulated tissues that was bordered by normal liver tissue (Fig. 2C and D).

Sizes of ablation lesions created by conventional RFA, NS-RFA and HS-augmented-RFA. Ablation lesions (Table I) 

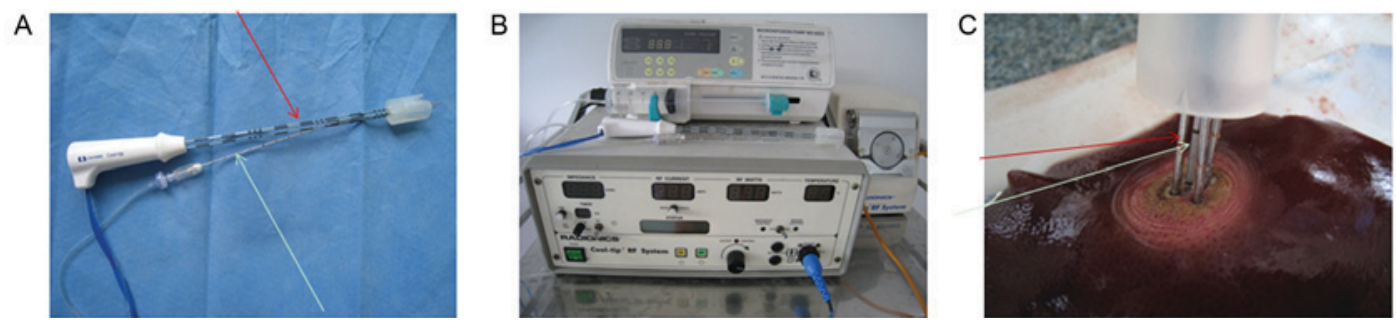

Figure 1. RF applicator. The instrument comprises (A) RF probes and (B) an RF generator. (C) A high-resolution image of 3 cool-tip electrodes (red arrow) and 1 perfused hypertonic-saline-augmented needle (white arrow) outlining the infusion system. RF, radiofrequency.

A

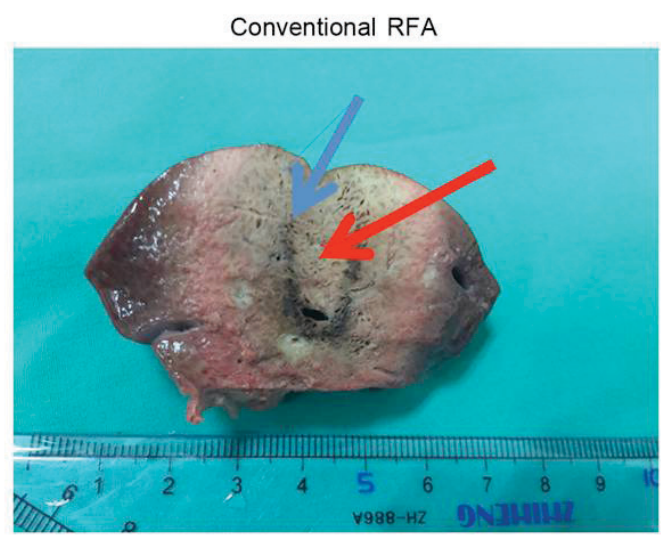

C

$6 \%$ Perfused hypertonic-saline-augmented-RFA

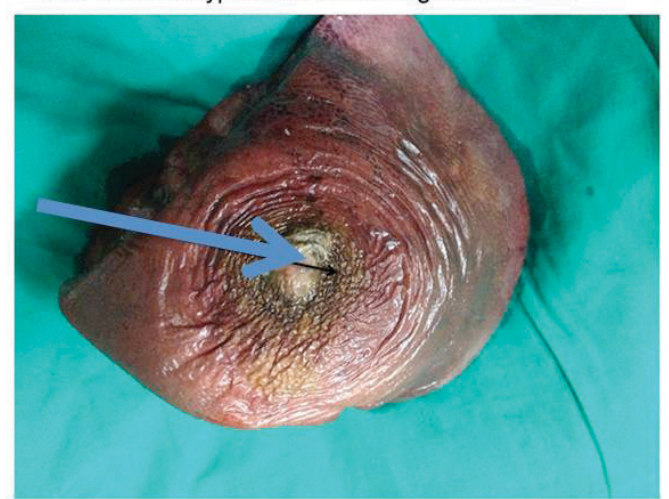

B

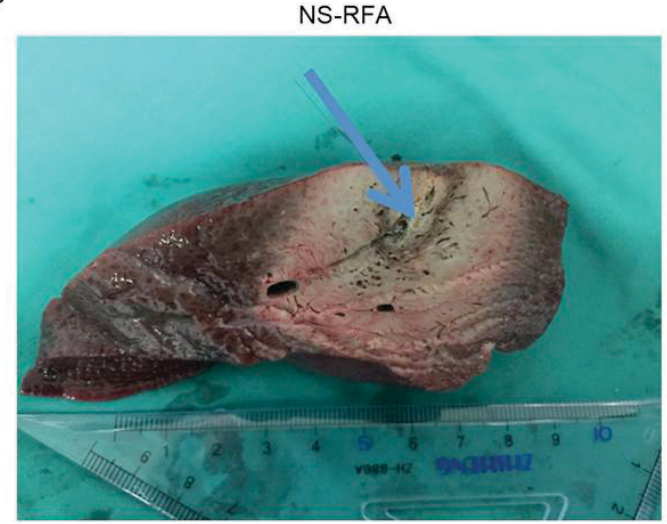

D

$6 \%$ Perfused hypertonic-saline-augmented-RFA

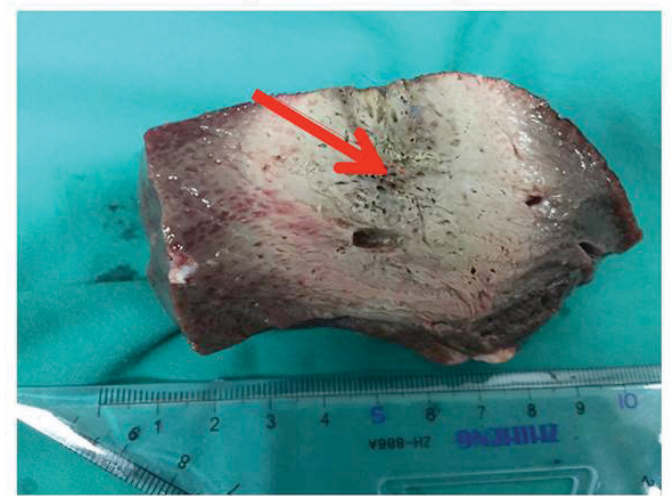

Figure 2. Macroscopic features of the 3 types of ablation lesions. An elliptical ablation lesion $(\sim 3.18 \times 2.27 \mathrm{~cm})$ was produced with conventional RFA. (A) A central hollow space along the electrode was surrounded by a lesion of pale coagulated tissue and a lesion of brownish tissue (red arrow). The blue arrow indicates the electrode track. (B) An elliptical-like ablation lesion $(\sim 3.85 \times 3.19 \mathrm{~cm})$ was produced with NS-RFA. The central cavity indicates the track of the channel and needle as they were withdrawn (blue arrow), surrounded by a lesion of pale tissue and a lesion of dust-colored tissue. (C) An elliptical-like ablation lesion $(\sim 4.67 \times 3.81 \mathrm{~cm})$ was produced with $6 \%$ perfused hypertonic-saline-augmented-RFA; the central portion of the lesion was jelly-like (blue arrow), and surrounded by a lesion of off-white tissue and a lesion of reddish tissue. (D) When the ablation lesion was cut perpendicularly to the needle, the central jelly-like portion displayed liquefaction necrosis, while the outer brown portion (red arrow) represented congestion and bleeding, such as edema. Blue arrow indicates the electrode track. NS, normal saline; RFA, radiofrequency ablation.

created by $6 \%$ perfused HS-augmented RFA were significantly larger compared with those created by conventional RFA or NS-RFA $(\mathrm{P}<0.05)$ (Fig. 3). Oval-shaped ablation zones were created with a larger transverse diameter at the midpoint compared with either the conventional or NS-RF application. Specifically, the mean volumes (Fig. 3C) of $8.99 \pm 0.52,21.79 \pm 1.05$ and $40.01 \pm 2.86 \mathrm{~cm}^{3}$ were obtained for ablation lesions created by conventional RFA, NS-RFA and HS-augmented-RFA, respectively. Concordantly, longitudinal (Fig. 3A) and transverse (Fig. 3B) diameters were significantly different in the three treatments groups (conventional RFA, $3.22 \pm 0.06$ and $2.31 \pm 0.04 \mathrm{~cm}$, respectively; NS-RFA, $4.13 \pm 0.08$ and $3.17 \pm 0.05 \mathrm{~cm}$, respectively; HS-augmented-RFA, $5.05 \pm 0.12$ and $3.89 \pm 0.09 \mathrm{~cm}$, respectively).

Histopathological data. Microscopically, the conventional-RFA lesions demonstrated centralized, complete destruction of the parenchyma. Coagulation necrosis created by conventional RFA revealed a zone of altered cellular morphology, which was best characterized as a heat effect that consisted of degenerated shrunken hepatocytes with pyknotic nuclei of the liver tissue. This region did not meet the classical criteria of coagulation necrosis defined by previous studies (19-21), but the cells were clearly different from normal 
Table I. Dimensions and volume of ablation lesions created in excised porcine livers by conventional RFA, NS-RFA and 6\% perfused hypertonic-saline-augmented-RFA.

\begin{tabular}{lccc}
\hline Dimension & Conventional RFA & NS-RFA & 6\% Perfused HS-augmented-RFA \\
\hline Longitudinal diameter, cm & $3.22 \pm 0.06$ & $4.13 \pm 0.08$ & $5.05 \pm 0.122$ \\
Transverse diameter, cm & $2.31 \pm 0.04$ & $3.17 \pm 0.05$ & $3.89 \pm 0.09$ \\
Volume, $\mathrm{cm}^{3}$ & $8.99 \pm 0.52$ & $21.79 \pm 1.05$ & $40.01 \pm 2.86$ \\
\hline
\end{tabular}

Data are presented as the mean \pm standard deviation and assessed via Mann Whitney U test. NS, normal saline; HS, hypertonic saline; RFA, radiofrequency ablation.
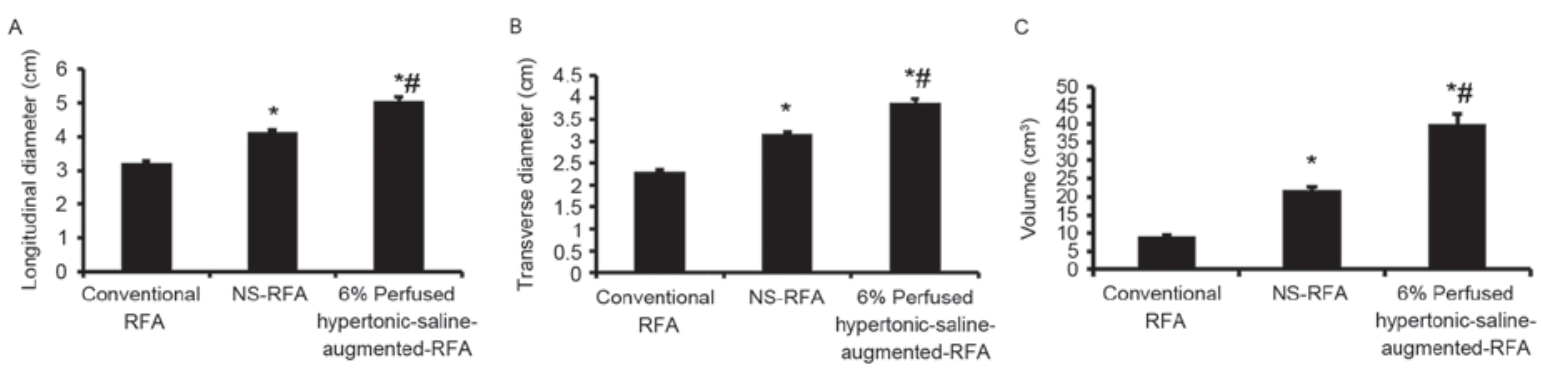

Figure 3. Sizes of ablation lesions created in excised porcine livers by conventional RFA, NS-RFA and $6 \%$ perfused hypertonic-saline-augmented-RFA according to (A) longitudinal diameter, (B) transverse diameter and (C) volume. " $\mathrm{P}<0.05$ vs. conventional-RFA group; ${ }^{*} \mathrm{P}<0.05$ vs. NS-RFA group. NS, normal saline; RFA, radiofrequency ablation.

hepatocytes. There was no marked difference between ablated lesions and normal liver tissue (Fig. 4A).

The coagulation lesions created by NS-RFA were similar to those of conventional RFA, but demonstrated a small blank area (central charred zone) where tissue was necrotic. Surrounding the central portion was coagulation necrosis and a peripheral hemorrhagic rim. Coagulation necrosis created by NS-RFA also appeared as irregular, degenerated and shrunken hepatic cells with pyknotic nuclei. However, the extent of hepatocyte damage in the lesions induced by NS-RFA was more substantial compared with the lesions produced by conventional RFA, and there was a marked difference between ablated lesions and normal liver tissue (Fig. 4B).

By contrast, the damage of liver cells created by $6 \%$ perfused HS-augmented-RFA was more severe compared with that induced by conventional RFA or NS-RFA. The central jelly-like tissue appeared as a homogeneous red dye-like substance, and coagulation necrosis in the outer layer of $6 \%$ perfused HS-augmented-RFA lesions was similar to that in NS-RFA lesions. A marked difference was observed between the ablated lesions and normal liver tissue (Fig. 4C). The normal liver H\&E staining images were used as controls (Fig. 4D).

\section{Discussion}

In the present study, multipolar RFA with $6 \% \mathrm{NaCl}$ demonstrated improved efficacy in creating a larger ablation zone compared with the conventional and $0.9 \% \mathrm{NaCl}$ modes.

In total, $70-85 \%$ of patients with liver cancer are not candidates for surgery (19-21). RFA destroys biological tissues with electromagnetic waves at frequencies between 460 and
$500 \mathrm{kHz}$, which is a high enough range to result in molecular frictional heating without stimulating a neuromuscular reaction and electrolysis, and which is low enough to confine the transmission of energy to a tissue mass in a more controlled manner, without excessive radiation being created $(22,23)$.

The major limitation of this approach is reaching an acceptable coagulation size (24). Also, abutting large blood vessels ( $\geq 3 \mathrm{~mm}$ ) reduces the effectiveness of the heat produced by RF due to perfusion-mediated tissue cooling within the area to be ablated, which consequently lowers RFA efficacy (25). The development of a number of strategies has improved tissue-energy interactions for thermal ablation therapy by increasing the region of induced coagulation $(11,13,26)$. One of the most effective approaches is the injection of saline or HS into the tissue during RFA via an electrode, which increases electrical conductance and thermal conductivity. The greater saline conductivity confers less resistive heating around the electrode tip $(11,26)$.

Lee et al (27) suggested that bipolar RFA resulted in larger, short-axis diameters of coagulation necrosis with $6 \% \mathrm{NaCl}$ solution compared with those created with $0.9 \% \mathrm{NaCl}$ solution. However, the results of the present study demonstrated that $\mathrm{NaCl}$ solution concentrations of $>6 \%$ did not additionally increase the extent of coagulation necrosis. In addition, $6 \%$ $\mathrm{NaCl}$ administered at a rate of $1.0 \mathrm{ml} / \mathrm{min}$ in bipolar RFA yielded larger necrosis diameters compared with values obtained with $0.5 \mathrm{ml} / \mathrm{min}$ (data not shown). In the present study, $\mathrm{NaCl}$ solution was introduced into the area of ablation during RFA using three-polar perfusion electrodes to enlarge the ablation volume, which achieved safe thermal ablation. The results demonstrated that $6 \%$ perfused HS-augmented-RFA lesions were significantly larger compared with the $0.9 \%$ NS-RFA or 


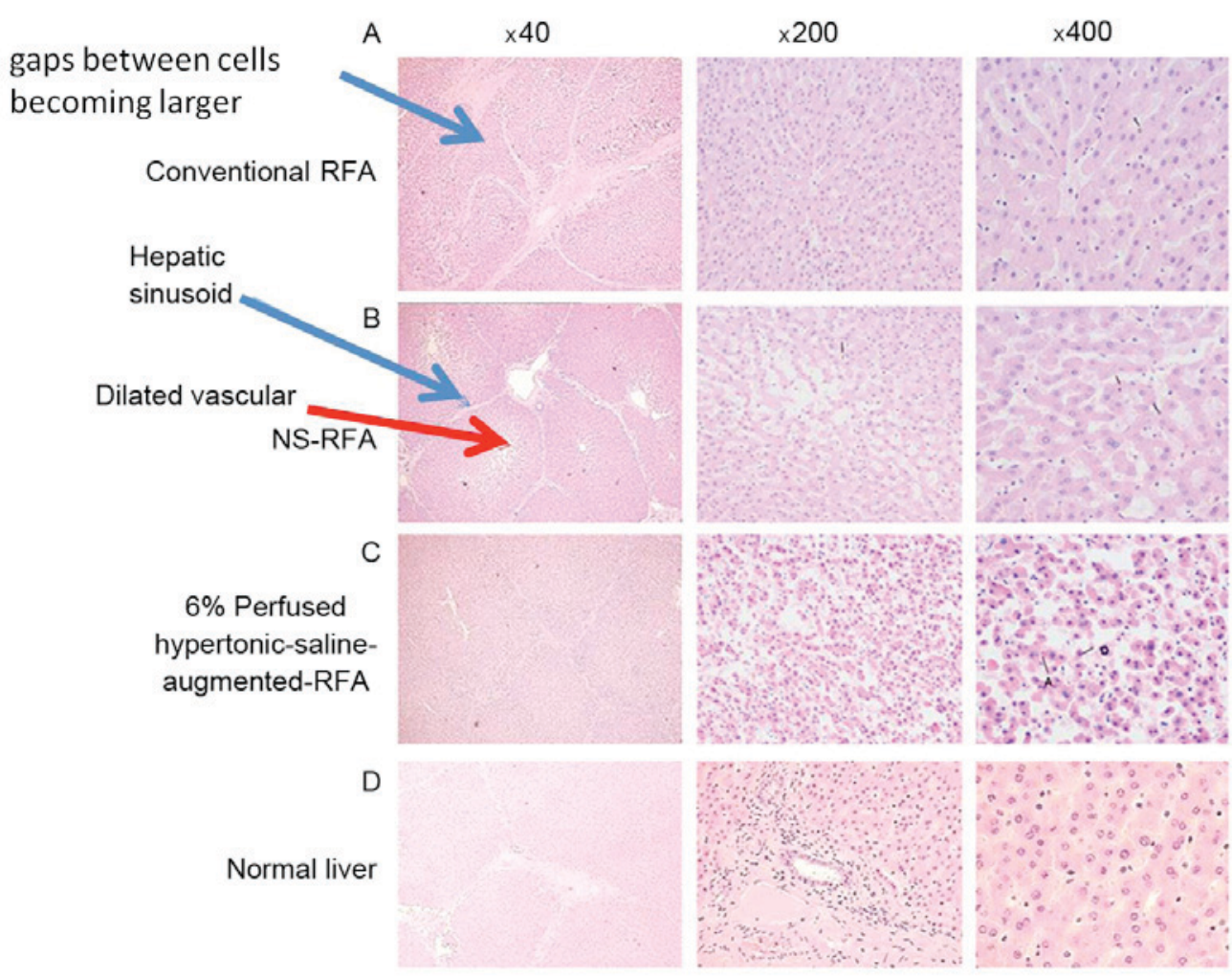

Figure 4. Micrographs of the coagulated ablation lesions created by conventional RFA, NS-RFA and 6\% perfused hypertonic-saline-augmented-RFA. (A) Left panel (H\&E; magnification, $\mathrm{x} 40$ ) demonstrates relatively sparse hepatocytes, with gaps between cells becoming larger. Liver borders and hepatic sinusoid remained normal, with dilated vessels. Central (H\&E; magnification, x200) and right (H\&E; magnification, x400) panels indicate coagulation necrosis and degenerated shrunken hepatocytes with pyknotic nuclei. (B) Left panel (H\&E; magnification, $\mathrm{x} 40$ ), morphology was slightly altered but normal cells were observed, similar to the control cells. Liver borders were ruptured, with hepatic sinusoid becoming sparse (blue arrow), larger gaps between cells and dilated vessels (red arrow). Central (H\&E; magnification, x200) and right (H\&E; magnification, x400) panels demonstrate more degenerated shrunken hepatocytes appearing with pyknotic nuclei compared with that demonstrated by the conventional and NA-RFA groups. Inflammatory cells and focal infiltration were observed. Red blood cells were also observed in the hepatic sinusoid. (C) Left panel (H\&E; magnification, $\mathrm{x} 40$ ) demonstrates damage to or disappearance of the structure of the liver borders and hepatic sinusoid. Hepatocytes are arranged in a disorderly manner. Central (H\&E; magnification, x200) and right (H\&E; magnification, $\mathrm{x} 400$ ) panels demonstrate the majority of hepatocytes becoming necrotic, deformed or ruptured. Apoptosis of hepatocytes was observed. Degenerated shrunken hepatocytes appeared with pyknotic nuclei and cytoplasmic condensation. Hepatocyte spacing was not evident. (D) Micrographs of the normal liver (H\&E; magnifications, x40, x200 and x400, respectively). NS, normal saline; RFA, radiofrequency ablation; H\&E, hematoxylin and eosin; NA-RFA, $0.9 \%$ saline-enhanced RFA group.

conventional RFA lesions. The most important factor that may explain this difference is that the ionic concentration of $6 \%$ perfused HS-augmented-RFA is much higher compared with that of NS $(0.9 \%)$, and therefore, the perfusion of the solution of $\mathrm{NaCl}$ during $6 \%$ perfused HS-augmented-RFA potentially increased the electrical conductivity of the tissue. Therefore, compared with NS-RFA, 6\% perfused HS-augmented-RFA may have created increased molecular friction, which could generate more energy and enlarge the ablated volume. In the pre-experiment of the present study, it was observed that $\mathrm{NaCl}$ concentrations $>6 \%$ do not enlarge the ablated lesions. This confirms the results identified by Lee et al (27). Although it has been demonstrated that hydrochloric acid RFA may be used to produce a larger ablated lesion size, the safety of diluted hydrochloric acid remains a concern (27). Solutions of $6 \%$ perfused $\mathrm{HS}$-augmented $\mathrm{NaCl}$ are much safer compared with using diluted hydrochloric acid, and $\mathrm{NaCl}$ does not require additional costs or medical fees.

Several studies have indicated that following RFA, the tissue appears to be almost unchanged microscopically $(23,28)$. Thermal coagulation necrosis induced by RFA differs from classic coagulation necrosis in that cells retain their original shape and the nuclei do not change, which makes it difficult to visualize by H\&E staining. In fact, these results were described in the literature as a 'ghost phenomenon' and cells exhibiting thermal coagulation necrosis were referred to as 'ghost cells' $(23,29-31)$. Nicotinamide adenine dinucleotide (NADH) vital staining may be used to identify the viability of hepatocytes with normal morphology $(23,31,32)$. However, NADH vital staining is more complicated than H\&E staining. In the present study, using H\&E staining with careful observation, it was identified that the majority of hepatocytes killed by NS-RFA appeared as coagulation necrosis microscopically. The results also demonstrated that $6 \%$ perfused HS-augmented-RFA produced more evident coagulation necrosis compared with NS-RFA. The coagulation necrosis created with $6 \%$ perfused HS-augmented-RFA was easily observed using H\&E staining, and therefore, NADH vital staining was not performed to identify cell viability.

A theoretical, yet unevaluated, oncological concern regarding the perfusion electrodes used in RFA is leakage from the electrode track of saline that is contaminated with viable tumor cells, resulting in peritoneal or track seeding. An additional uninvestigated concern is that the saline may increase the intra-tumoral 
pressure and force tumor cells into the circulation, thus resulting in lymphatic or hematogenous seeding $(23,33,34)$. However, $6 \%$ perfused HS-augmented-RFA may reduce these risks, as tumor cells cannot survive easily in this high concentration liquid; however, this requires additional confirmation.

Limitations of the present study include the fact that normal tissues in ex vivo porcine livers, and not in vivo or metastatic HCC tumor tissues, were used. Therefore, the distribution of infused liquid (NS or $6 \%$ perfused HS-augmented) differed due to the different tissue architecture. Also, without the cooling effect of blood flow, the ablated volume in ex vivo tissues may be larger compared with that produced in in vivo tissue. Additionally, other parameters of RFA treatment efficacy, such as tumor number and location, body tolerance to the procedure and associated complications (e.g., liver failure), were not assessed in the present ex vivo study. Therefore, the results are preliminary and provide a reference for future investigations. Although $6 \%$ perfused HS-augmented is safe and efficient when it is used in ablation, the safety of $6 \%$ perfused HS-augmented requires additional investigation, which will be addressed in a future study.

In conclusion, the present study demonstrated that $6 \%$ perfused HS-augmented-RFA creates larger ablation zones compared with either the conventional method or NS-RF application. These data suggest that $6 \%$ perfused HS-augmented-RFA may be superior to conventional or NS-RF applications, with the potential to improve the results of RFA for the treatment of larger tumors.

\section{References}

1. Ikeda K, Kobayashi M, Kawamura Y, Imai N, Seko Y, Hirakawa M, Hosaka T, Sezaki H, Akuta N, Saitoh S, et al: Stage progression of small hepatocellular carcinoma after radical therapy: Comparisons of radiofrequency ablation and surgery using the Markov model. Liver Int 31: 692-699, 2011.

2. van Duijnhoven FH, Jansen MC, Junggeburt JM, van Hillegersberg R, Rijken AM, van Coevorden F, van der Sijp JR, van Gulik TM, Slooter GD, Klaase JM, et al: Factors influencing the local failure rate of radiofrequency ablation of colorectal liver metastases. Ann Surg Oncol 13: 651-658, 2006.

3. Berber E and Siperstein A: Local recurrence after laparoscopic radiofrequency ablation of liver tumors: An analysis of 1032 tumors. Ann Surg Oncol 15: 2757-2764, 2008.

4. Goldberg SN, Gazelle GS, Dawson SL, Rittman WJ, Mueller PR and Rosenthal DI: Tissue ablation with radiofrequency using multiprobe arrays. Acad Radiol 2: 670-674, 1995.

5. Goldberg SN, Solbiati L, Hahn PF, Cosman E, Conrad JE, Fogle R and Gazelle GS: Large-volume tissue ablation with radio frequency by using a clustered, internally cooled electrode technique: Laboratory and clinical experience in liver metastases. Radiology 209: 371-379, 1998.

6. Goldberg SN, Gazelle GS, Dawson SL, Rittman WJ, Mueller PR and Rosenthal DI: Tissue ablation with radiofrequency: Effect of probe size, gauge, duration, and temperature on lesion volume. Acad Radiol 2: 399-404, 1995.

7. de Baere T, Denys A, Wood BJ, Lassau N, Kardache M, Vilgrain V, Menu Y and Roche A: Radiofrequency liver ablation: Experimental comparative study of water-cooled versus expandable systems. AJR Am J Roentgenol 176: 187-192, 2001.

8. Solazzo SA, Ahmed M, Liu Z, Hines-Peralta AU and Goldberg SN: High-power generator for radiofrequency ablation: Larger electrodes and pulsing algorithms in bovine ex vivo and porcine in vivo settings. Radiology 242: 743-750, 2007.

9. Lorentzen T: A cooled needle electrode for radiofrequency tissue ablation: Thermodynamic aspects of improved performance compared with conventional needle design. Acad Radiol 3: $556-563,1996$.
10. Luo RG, Gao F, Gu YK, Huang JH and Li CL: Radioablation settings affecting the size of lesions created ex vivo in porcine livers with monopolar perfusion electrodes. Acad Radiol 17: 980-984, 2010.

11. Rempp H, Voigtländer M, Clasen S, Kempf S, Neugebauer A Schraml C, Schmidt D, Claussen CD, Enderle MD, Goldberg SN and Pereira PL: Increased ablation zones using a cryo-based internally cooled bipolar RF applicator in ex vivo bovine liver. Invest Radiol 44: 763-768, 2009.

12. Burdio F, Güemes A, Burdío JM, Navarro A, Sousa R, Castiella T, Cruz I, Burzaco O, Guirao X and Lozano R: Large hepatic ablation with bipolar saline-enhanced radiofrequency: An experimental study in in vivo porcine liver with a novel approach. J Surg Res 110: 193-201, 2003.

13. Gangi A, Guth S and Imbert J: Interest of radiofrequency liver tissue ablation with a bipolar-wet electrode. Eur Radiol 133: 477, 2003.

14. Lee JM, Han JK, Kim SH, Sohn KL, Lee KH, Ah SK and Choi BI: A comparative experimental study of the in-vitro efficiency of hypertonic saline-enhanced hepatic bipolar and monopolar radiofrequency ablation. Korean J Radiol 4: 163-169, 2003.

15. Schmidt D, Trübenbach J, Brieger J, Koenig C, Putzhammer H, Duda SH,Claussen CD and Pereira PL: Automated saline-enhanced radiofrequency thermal ablation: Initial results in ex vivo bovine livers. AJR Am J Roentgenol 180: 163-165, 2003.

16. Hänsler J, Frieser M, Schaber S, Kutschall C, Bernatik T, Müller W, Becker D, Hahn EG and Strobel D: Radiofrequency ablation of hepatocellular carcinoma with a saline solution perfusion device: A pilot study. J Vasc Interv Radiol 14: 575-580, 2003.

17. Lee JM, Kim SH, Han JK, Sohn KL and Choi BI: Ex vivo experiment of saline-enhanced hepatic bipolar radiofrequency ablation with a perfused needle electrode: Comparison with conventional monopolar and simultaneous monopolar modes. Cardiovasc Intervent Radiol 28: 338-345, 2005.

18. Seong NJ, Yoon CJ, Kang SG, Chung JW, Kim HC and Park JH: Effects of arsenic trioxide on radiofrequency ablation of VX2 liver tumor: Intraarterial versus intravenous administration. Korean J Radiol 13: 195-201, 2012.

19. McWilliams JP, Yamamoto S, Raman SS, Loh CT, Lee EW, Liu DM and Kee ST: Percutaneous ablation of hepatocellular carcinoma: Current status. J Vasc Interv Radiol 21 (8 Suppl): S204-S213, 2010.

20. Rhim H, Choi D, Kim YS, Lim HK and Choe BK: Ultrasonography-guided percutaneous radiofrequency ablation of hepatocellular carcinomas: A feasibility scoring system for planning sonography. Eur J Radiol 75: 253-258, 2010.

21. Garrean S, Hering J, Saied A, Helton WS and Espat NJ: Radiofrequency ablation of primary and metastatic liver tumors: A critical review of the literature. Am J Surg 195: 508-520, 2008.

22. Shiina S: Image-guided percutaneous ablation therapies for hepatocellular carcinoma. J Gastroenterol 44 (Suppl 19): S122-S131, 2009.

23. Nahum Goldberg S and Dupuy DE: Image-guided radiofrequency tumor ablation: Challenges and opportunities-part I. J Vasc Interv Radiol 12: 1021-1032, 2001.

24. Ni Y, Mulier S, Miao Y, Michel L and Marchal G: A review of the general aspects of radiofrequency ablation. Abdom Imaging 30: 381-400, 2005.

25. Bruners P, Pfeffer J, Kazim RM, Günther RW, Schmitz-Rode T and Mahnken AH: A newly developed perfused umbrella electrode for radiofrequency ablation: An ex vivo evaluation study in bovine liver. Cardiovasc Intervent Radiol 30: 992-998, 2007.

26. Tamaki K, Shimizu I, Oshio A, Fukuno H, Inoue H, Tsutsui A, Shibata H, Sano N and Ito S: Influence of large intrahepatic blood vessels on the gross and histological characteristics of lesions produced by radiofrequency ablation in a pig liver model. Liver Int 24: 696-701, 2004.

27. Lee JM, Kim YK, Lee YH, Kim SW, Li CA and Kim CS: Percutaneous radiofrequency thermal ablation with hypertonic saline injection: In vivo study in a rabbit liver model. Korean J Radiol 4: 27-34, 2003.

28. Luo RG, Fao F, Huang JH, Gu YK, Jiang XY and Huang YJ: Diluted hydrochloric acid generates larger radiofrequency ablation lesions in excised porcine livers. Diagn Interv Radiol 19: 145-149, 2013.

29. Miao Y, Ni Y, Mulier S, Yu J, De Wever I, Penninckx F, Baert AL and Marchal G: Treatment of VX2 liver tumor in rabbits with 'wet' electrode mediated radio-frequency ablation. Eur Radiol 10: 188-194, 2000. 
30. Goldberg SN, Girnan GD, Lukyanov AN, Ahmed M Monsky WL, Gazelle GS, Huertas JC, Stuart KE, Jacobs T, Torchillin VP, et al: Percutaneous tumor ablation: Increased necrosis with combined radio-frequency ablation and intravenous liposomal doxorubicin in a rat breast tumor model. Radiology 222: 797-804, 2002.

31. Hänsler J, Neureiter D, Wasserburger M, Janka R, Bernatik T, Schneider T, Müller W, Frieser M, Schaber S, Becker D, et al: Percutaneous US-guided radiofrequency ablation with perfused needle applicators: Improved survival with the VX2 tumor model in rabbits. Radiology 230: 169-174, 2004.

32. Shen P, Geisinger KR, Zagoria R and Levine EA: Pathologic correlation study of microwave coagulation therapy for hepatic malignancies using a three-ring probe. J Gastrointest Surg 11: 603-611, 2007.
33. Simon CJ, Dupuy DE, Iannitti DA, Lu DS, Yu NC, Aswad BI, Busuttil RW and Lassman C: Intraoperative triple antenna hepatic microwave ablation. AJR Am J Roentgenol 187: W333-W340, 2006.

34. Ni Y, Miao Y, Mulier S, Yu J, Baert AL and Marchal G: A novel 'cooled-wet' electrode for radiofrequency ablation. Eur Radiol 10: 852-854, 2000.

(1) This work is licensed under a Creative Commons Attribution-NonCommercial-NoDerivatives 4.0 International (CC BY-NC-ND 4.0) License. 